\title{
Gravitational lens surveys with LOFAR
}

\author{
Olaf Wucknitz ${ }^{* \dagger}$ \\ Argelander-Institut für Astronomie, Auf dem Hügel 71, 53121 Bonn, Germany \\ Joint Institute for VLBI in Europe, Oude Hoogeveensedijk 4, 7991 PD Dwingeloo, The \\ Netherlands \\ E-mail: wucknitz@astro.uni-bonn.de
}

\section{Garrett}

ASTRON, Oude Hoogeveensedijk 4, 7991 PD Dwingeloo, The Netherlands

Deep surveys planned as a Key Science Project of LOFAR provide completely new opportunities for gravitational lens searches. For the first time do large-scale surveys reach the resolution required for a direct selection of lens candidates using morphological criteria. We briefly describe the strategies that we will use to exploit this potential. The long baselines of an international $E$-LOFAR are essential for this project.

From planets to dark energy: the modern radio universe October 1-5 2007

University of Manchester, Manchester, UK

* Speaker.

$\dagger$ This work is supported by the European Community’s Sixth Framework Marie Curie Research Training Network Programme, Contract No. MRTN-CT-2004-505183 "ANGLES", and by the Emmy-Noether-Programme of the 'Deutsche Forschungsgemeinschaft', reference WU 588/1-1. 


\section{Introduction}

Gravitational lensing is the best method to determine the mass distribution of distant galaxies with high accuracy. With a large sample of lens systems at a range of redshifts, we can study not only the structure but also the evolution of galaxies. We identify three main topics, in which lensing can help to resolve controversial problems.

- Most galaxies at moderate redshifts seem to have very close to isothermal $\left(\rho \propto r^{-2}\right)$ mass distributions. Why is this the case, and does it also hold for higher redshifts?

- How does the central density profile of galaxies look like? Do they have cores or cusps, how do masses of central black holes evolve?

- Are small sub-halos as abundant as predicted by the CDM structure formation scenario?
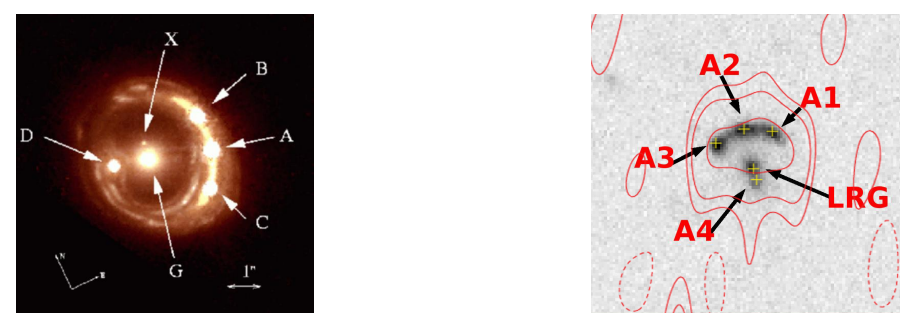

Figure 1: Two examples from the bright end of typical lens systems expected to be found in LOFAR surveys. Left: RXS J1131-1231 (HST image from Claeskens et al., 2006). Right: The ' 8 o'clock arc' (Allam et al., 2007). WSRT $5 \mathrm{GHz}$ contours superimposed upon SDSS image.

We can help answering these questions by modelling the mass distributions to fit for the structure of gravitationally lensed sources. In order to obtain information about the complete mass distribution, lensed extended sources like the ones shown in Fig. 1 should be used and modelled with LensClean (Wucknitz, 2004). There are several advantages in using radio observations. Most important are the wide range of resolutions that can be achieved with radio arrays and the absence of effects like dust extinction and microlensing. So far we know less than 50 radio lenses, whilst the number of optical lenses has grown well above that and is increasing further.

\section{New lens surveys}

\subsection{Source-targeted search}

LOFAR not only probes a new frequency range but also new parameter space in terms of resolution and sensitivity of wide-area radio surveys. With these data, it is for the first time possible to identify lens systems directly from the source surveys using morphological criteria. Following up complete source samples as done in CLASS (Myers et al., 2003; Browne et al., 2003) would be prohibitive for the extreme number of sources expected in LOFAR surveys.

The expected lensing rates for this source population is 1:2000, leading to potential numbers of 400000 and 15000 lenses in the LOFAR surveys at 120 and $200 \mathrm{MHz}$, respectively. Because of sensitivity and resolution limitations, we will be able to identify only a small fraction of these 

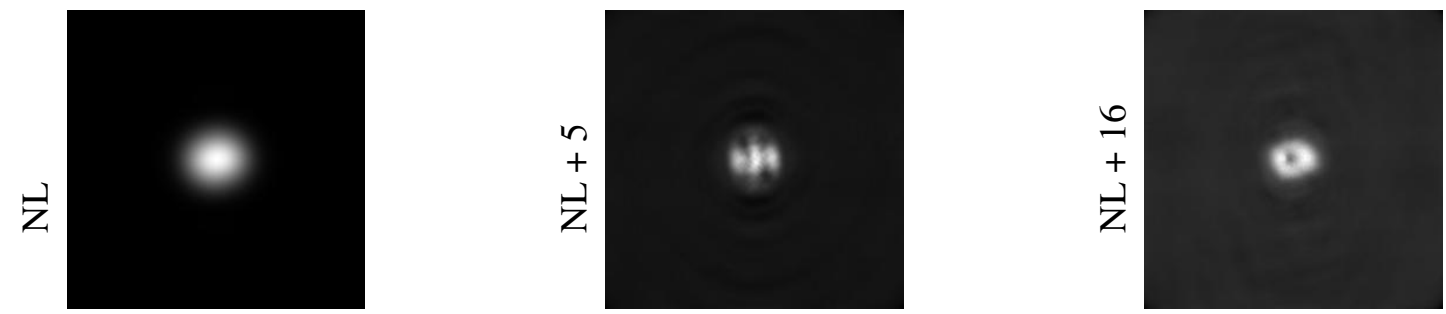

Figure 2: Simulated Clean maps of a lensed star-burst galaxy with three different LOFAR arrays. The full international LOFAR is needed to properly resolve and map the lensed source.

lenses. At $200 \mathrm{MHz}, 900$ lenses are a more realistic number, for which we probably have to followup around 10000 candidates with the EVLA and/or $e$-MERLIN. At $120 \mathrm{MHz}$, longer baselines would be needed to detect more than just the systems with the largest image separations.

A good fraction of the LOFAR sources will be star-bursts galaxies, and the majority will have extensions and structures exactly on the scale that is needed for accuracte lens models. We can thus build the perfect sample for the study of the structure and evolution of galaxies.

\subsection{Lens-targeted search}

An alternative strategy to find lens systems is to add the information from optical galaxy catalogues listing potential lensing galaxies at moderate redshifts. By cross-correlating these galaxy catalogues with the LOFAR source catalogues, we can establish a large sample of lens systems with well-defined selection criteria for the lens galaxies. Combining the Luminous Red Galaxies (LRG) from the SDSS with the $120 \mathrm{MHz}$ LOFAR survey, we expect to find about 500 lenses in 5000 candidates. With upcoming larger galaxy surveys, this number can be significantly increased.

\section{Limitations}

Even with additional stations in neighbouring countries, the resolution of LOFAR will be sufficient to securely identify only lenses with relatively large separations. Fig. 2 shows simulations of a lensed star-burst galaxy observed with three different arrays. We see that a good number of international stations is not only required for the resolution but also for good mapping properties.

The recently announced de-scope of LOFAR will modify the expectations for the lens surveys. Very roughly, a drop in sensitivity by a factor of 2 will lead to a reduction in the number of lenses by the same factor. Realistic expectations will depend on the adapted survey strategy and the number of international stations, which can partly compensate for the reduction of Dutch LOFAR.

\section{References}

Allam, S. S., Tucker, D. L., Lin, H., et al. 2007, ApJ, 662, L51

Browne, I. W. A., Wilkinson, P. N., Jackson, N. J. F. ., et al. 2003, MNRAS, 341, 13

Claeskens, J.-F., Sluse, D., Riaud, P., \& Surdej, J. 2006, A\&A, 451, 865

Myers, S. T., Jackson, N. J., Browne, I. W. A., et al. 2003, MNRAS, 341, 1

Wucknitz, O. 2004, MNRAS, 349, 1 